\title{
Frequency and component analysis of contaminants generated in preparation of anticancer agents using Closed system drug transfer devices (CSTDs)
}

\section{Satomi Sumikawa}

Ehime University Hospital

Yoshihiro Yakushijin ( $\nabla$ yoshiyak@m.ehime-u.ac.jp)

Ehime University Graduate School of Medicine

Kenjiro Aogi

NHO Shikoku Cancer Center

Takuya Yano

Sumitomo-Besshi Hospital

Hiroki Hashimoto

Matsuyama Red Cross Hospital

Chiyuki Tsukui

Matsuyamashimin Hospital

Tadashi Noguchi

Saiseikai Imabari Hospital

\section{Taro Shiraishi}

Shikoku Central Hospital of the Mutual aid Association of Public School teachers

\section{Yasuhiro Horikawa}

Jyuzen General Hospital

\section{Yasuo Yasuoka}

Saiseikai Matsuyama Hospital

Akihiro Tanaka

Saiseikai Saijo Hospital

Noriaki Hidaka

Ehime University Hospital

Mamoru Tanaka

Ehime University Hospital 
Keywords: anticancer agents, preparation, closed system drug transfer devices (CSTDs), contaminants Posted Date: April 21st, 2021

DOI: https://doi.org/10.21203/rs.3.rs-418319/v1

License: (c) (1) This work is licensed under a Creative Commons Attribution 4.0 International License. Read Full License

Version of Record: A version of this preprint was published at Scientific Reports on January 7th, 2022. See the published version at https://doi.org/10.1038/s41598-021-03780-0. 
Frequency and component analysis of contaminants generated in preparation of anticancer agents using Closed system drug transfer devices (CSTDs)

Satomi Sumikawa ${ }^{1}$, Yoshihiro Yakushijin ${ }^{2 *}$, Kenjiro Aogi ${ }^{3}$, Takuya Yano ${ }^{4}$, Hiroki Hashimoto ${ }^{5}$, Chiyuki Tsukui ${ }^{6}$, Tadashi Noguchi ${ }^{7}$, Taro Shiraishi $^{8}$, Yasuhiro Horikawa $^{9}$, Yasuo Yasuoka ${ }^{10}$, Akihiro Tanaka $^{11}$, Noriaki Hidaka ${ }^{1}$ and Mamoru Tanaka ${ }^{1}$

1 Division of Pharmacy, Ehime University Hospital, Ehime, 7910295, Japan

2 Department of Clinical Oncology, Ehime University Graduate School of Medicine, Ehime, 7910295, Japan

3 Division of Clinical Research Promotion, NHO Shikoku Cancer Center, Ehime, 7910280, Japan

4 Division of Pharmacy, Sumitomo-Besshi Hospital, Ehime, 7928543, Japan

5 Department of Pharmacy, Matsuyama Red Cross Hospital, Ehime, 7908524, Japan

6 Division of Pharmacy, Matsuyamashimin Hospital, Ehime, 7900067, Japan

7 Department of Pharmacy, Saiseikai Imabari Hospital, Ehime, 7991592 , Japan 
8 Department of Pharmacy, Shikoku Central Hospital of the Mutual aid Association of

Public School teachers, Ehime, 7990193, Japan

9 Jyuzen General Hospital, Ehime, 7928586, Japan

10 Saiseikai Matsuyama Hospital, Ehime, 7918026, Japan

11 Division of Pharmacy, Saiseikai Saijo Hospital, Ehime, 7930027, Japan

*Correspondonding author. Yoshihiro Yakushijin M.D., Ph.D.

Department of Clinical Oncology,

Ehime University Graduate School of Medicine,

Shitsukawa, Toon, Ehime 7910295, Japan

Tel: +81-89-960-5296. Fax: +81-89-960-5969. E-mail: yoshiyak@m.ehime-u.ac.jp 


\section{Abstract}

Occupational exposure of anticancer agents during their preparation has been recognized as a serious hazard. Closed system drug transfer devices (CSTDs) enable "safe" preparation of agents for medical personnel and ensure a safe hospital environment. However, artificial particles of infusion materials have been reported during CSTD use. Here, the incidence of insoluble fine particles during preparation of anticancer agents using CSTDs was examined. Visible insoluble fine particles were found in 465 (9.4\%) of 4,948 treatment cases at Ehime University Hospital with CSTD use. Contaminants occurred more frequently during preparation of monoclonal antibodies than cytotoxic anticancer agents $(19.4 \%$ versus $4.1 \%$, respectively, $\mathrm{P}<0.01)$. A similar survey was conducted at nine hospitals to investigate the incidence of insoluble fine particles with or without CSTDs. Insoluble fine particles were detected in 113 (15.4\%) of 732 treatment cases during preparation of monoclonal antibodies with CSTD use. In contrast, the occurrence of insoluble fine particles without CSTDs was found in only $3(0.073 \%)$ of 4,113 treatment cases. Contamination with CSTDs might cause harmful effects on patients 
during cancer therapy. We strongly recommend the use of in-line filters combined with infusion routes after CSTD use to avoid contamination-associated adverse events. 


\section{Introduction}

Intravenous injectables consist of solution-containing therapeutic agents. However, endogenous artificial foreign substances may be introduced during several steps of manufacturing procedures by contaminated equipment or environmental sources. These foreign substances are designated as "insoluble foreign matters" or "insoluble fine particles". ${ }^{1}$ Insoluble foreign matters and insoluble fine particles are distinguished by size. Insoluble foreign matters are recognized as visible foreign contaminants of solutions that can be easily removed from materials. In contrast, very fine substances recognized with careful observation and scientific analysis are designated as insoluble fine particles. These fine particles are sometimes difficult to remove from materials during medical procedures, and may consequently cause biological effects on patient health outside of the original medical treatment. For example, intravenous administration of insoluble fine particles and glass ampoules has been noted to cause damage to the vein, lung, liver, and spleen in rare cases..$^{2-8}$ Therefore, insoluble fine particles are potential health hazards and should be carefully removed from medical procedures in patients receiving long-term treatment, such as cancer patients. 
In recent cancer treatment, occupational exposure of anticancer agents during preparation has been recognized as a serious environmental problem of cancer treatment. ${ }^{9-17}$ Therefore, closed system drug transfer devices (CSTDs) were universally introduced to chemotherapy. ${ }^{18-22}$ These technological improvements and devices have enabled safe administration of anticancer agents not only for patients and medical personnel but also hospital environments. However, CSTDs contain multiple moving parts such as vapor-trapping pockets with needles or plastic spikes, syringe units, and lubricants. Therefore, CSTD use may increase contamination of intravenous injectables and consequently be a source of health hazards in cancer patients receiving long-term medical treatment. Here, we analyzed the frequency and components of insoluble fine particles generated during the preparation of anticancer agents using CSTDs in infusion devices and discussed the influence, prevention, and countermeasures for cancer treatment.

\section{Results}


Incidence of insoluble fine particles generated during preparation of anticancer agents

with CSTDs

In early 2018, some pharmacists noticed the existence of insoluble fine particles generated during preparation of anticancer agents with CSTDs. After checking for these contaminants, we examined the frequency of insoluble fine particles during preparation of each anticancer agent for 1 year (January 31, 2018-January 30, 2019). Table 1 shows the incidence of insoluble fine particles by drug type (monoclonal antibodies versus cytotoxic agents) because we observed that the incidence of insoluble fine particles during preparation of recently produced monoclonal antibodies appeared to be higher than that during the preparation of cytotoxic agents. Pembrolizumab (anti-PD-1 antibody) showed the highest incidence of contamination of insoluble fine particles, accounting for $62.5 \%$ of contaminants. Other monoclonal antibodies, such as ramucirumab (48\%), panitumumab (45\%), and mogamulizumab (40\%), also showed surprisingly higher incidents of insoluble fine particles compared to cytotoxic agents for cancer chemotherapies (Figure 1). 
Eight hospitals including our institute and one cancer center in Ehime Prefecture participated in the current study. Among these institutes, three institutes routinely used CSTDs for all anticancer drugs. Two institutes used CSTDs only for highly carcinogenic anticancer agents, and the remaining four institutes used CSTDs only for volatile anticancer agents (cyclophosphamide, ifosfamide, and bendamustine). The summary of the preparation of anticancer agents with or without CSTDs in the nine institutes is indicated in Table 2.

Table 3 summarizes the frequencies of visible contamination of insoluble fine particles. Insoluble fine particles were found in $16(0.17 \%)$ of 9,434 preparations without CSTD use. In contrast, insoluble fine particles were found in 63 (2.1\%) of 3,036 preparations with CSTD use. The incidence of visible contamination was significantly higher with CSTDs than without CSTDs $(P<0.01)$. These cases were examined by 104 pharmacists from all institutes included in the study to confirm the data (Table S1).

Verification of insoluble fine particles using SEM, FTIR, and SEM-EDS analysis

To verify the number of insoluble fine particles trapped in in-line filters after cancer chemotherapies, 13 in-line filters and 3 control filters were randomly selected and 
recovered. Insoluble fine particles trapped in in-line filters were observed with a microscope, and trapped components were analyzed by FTIR. Figure 2 shows representative photographs of insoluble fine particles, gray substances measuring approximately $100 \mu \mathrm{m}$, trapped in the in-line filter. FTIR analysis indicated that one gray particle (Figure 2A) was a polysaccharide containing a carboxylic acid structure. Another gray particle (Figure 2B) consisted of polypropylene as the main component, suggesting that this was a fatty acid component. The structure of the last particle (Figure 2C) seemed to be polypropylene, suggesting that this was silicone as an accessory component in CSTDs. From these analyses, we decided to implement routine use of inline filters into infusion routes to avoid biological damage from insoluble fine particles during cancer treatment. The possibility of other contaminants and debris, such as rubbers, plastic utensils, and needle shards, could not be completely denied from past reports. ${ }^{23-25}$

\section{Discussion}


Our current study detected three important findings. First, the incidence of insoluble fine particles in cases using CSTDs during cancer chemotherapies was significantly higher than in those not using CSTDs. Second, the incidence of insoluble fine particles was significantly higher in cases treated with monoclonal antibodies than in those treated with other cytotoxic anticancer agents. Third, the incidence of insoluble fine articles varies among medical institutes.

The risk of contamination of insoluble fine particles in injection materials has been noted and was discussed in the early 1960s. ${ }^{23}$ The British Pharmacopoeia subsequently announced and established regulations on insoluble fine particles in injection materials in 1973, and the United States Pharmacopeia also established similar regulations in 1984. ${ }^{26,27}$ The criteria for insoluble fine particles for injectables have been described in the 14th revised Japanese Pharmacopoeia in 2001 to ensure the safety of injectables in Japan. ${ }^{28}$ Consequently, contamination of insoluble fine particles during manufacturing procedures is within the standard range. However, recent advances in cancer chemotherapies require complicated management of each agent, such as storage, dispensing, and administration, and several materials are used for preparation and 
administration during treatment. One of these complications is derived from CSTDassociated materials. A CSTD can prevent biohazards. However, CSTDs consists of several steps and related materials such as vapor-trapping pockets, needles or plastic spikes, syringe units, and lubricants. Our current observation study suggests that CSTD use may increase the number of contaminants in intravenous injectables and subsequently augment risks of health hazards during cancer chemotherapies.

Interestingly, we detected a higher incidence of insoluble fine particles in patients treated with monoclonal antibodies. Nakayama et al. ${ }^{29}$ reported several differences in solutions, coating materials of vials, and type (material and hardness) of rubber stoppers used as recent medical materials. In the current study, insoluble fine particles were more frequently generated in cases using CSTDs than in cases using conventional preparations (syringe and needle) without CSTDs. For example, a coating material such as silicone oil is applied to the inside of the syringe to improve slidability of the plunger. For low molecular weight compounds, application of silicone oil is not a major problem; however, for protein preparations, application of silicone oil may cause protein 
aggregation..$^{30,31}$ We speculate that this may explain the high incidence of contamination in cases treated with monoclonal antibodies.

We also noted differences in the incidence of contaminants among medical institutes. Since the first observation of insoluble fine particles during preparation of anticancer agents in early 2018, pharmacists at Ehime University Hospital have carefully checked for impurities. However, the incidence of contamination at our institute in this survey was extremely high $(9.2 \% ; 167$ CSTD cases of 1,818 preparations of anticancer agents at Ehime University Hospital) compared to those of other institutes $(0.4 \%$; 9 CSTD cases of 2,227 preparations of anticancer agents in 7 other institutes) (Table 3). This discrepancy may be partially explained by the number of patients receiving monoclonal antibody treatments and types of material used, such as CSTD, infusion route, needle, and syringe. However, we could not determine the precise reason for this difference in incidence.

Lastly, our analysis suggested that possible contaminants among insoluble fine particles might include polysaccharides, fatty acid components, and silicone, which are probably derived from coating materials of vials, syringes, and CSTDs and related materials. However, the number of samples examined by FTIR and SEM-EDS was limited. 
The possibility of other contaminants and debris, such as rubbers, plastic utensils, and needle shards, could not be completely denied. Furthermore, we observed oleamide crystals in infusion tubes (data not shown). Oleamide is used as a coating material for intravenous tubing and has no adverse effects in humans. However, the effect of oleamide crystals and long-term exposure in patients, especially cancer patients, is not fully understood, suggesting that it may be better to avoid this contaminant as much as possible.

Based on the findings of the current study, we strongly recommend the use of in-line filters to prevent artificial contamination in anticancer agents administered intravenously or monoclonal antibodies administered with CSTDs. Cancer treatments typically induce adverse events such as allergy, chills, and fever. However, some of these adverse events may be derived from artificial contamination during CSTD use, and these adverse events may be prevented by the routine application of in-line filters.

\section{Material and Methods}


Incidence of insoluble fine particles generated during preparation of anticancer agents using CSTDs at Ehime University Hospital

From January 31, 2018 to January 30, 2019 (1-year period), the frequency of insoluble fine particles discovered in the chemotherapy room for outpatients at Ehime University Hospital was aggregated by each anticancer agent. Insoluble fine particles were examined during preparation procedures for anticancer agents, such as in syringes adjusted with anticancer agents, vials after mixture, and infusion bags after CSTD use. Once insoluble fine particles were recognized at any step of the preparation procedure, an additional pharmacist confirmed the presence of insoluble fine particles. When multiple insoluble fine particles were observed during different preparation steps of one anticancer agent, the number of insoluble fine particles generated was counted as one. The incidence of insoluble fine particles divided by number of drug preparations, i.e., frequency/anticancer agent, was aggregated and statistically analyzed.

Comparison of frequency of insoluble fine particles with and without CSTD use at other hospitals in Ehime Prefecture 
Frequencies of contamination by insoluble fine particles in eight hospitals, including Ehime University Hospital, and one cancer center were investigated using the same method described above. Each institute evaluated the frequency of contamination over a consecutive 3-month period within January-June 2019, and the incidence of contamination of insoluble particles and CSTD types were compared.

\section{Verification of insoluble fine particles after CSTD use}

To avoid hazardous effects in cancer patients receiving chemotherapy after observation of insoluble fine particles with CSTD use, we applied in-line filters $(0.2 \mu \mathrm{m})$ to trap the impurities during chemotherapy starting from March 2018 in our institute. Therefore, we next attempted to verify insoluble particles to analyze the trapped materials.

After chemotherapy, in-line filters were randomly selected, recovered, and washed five times with distilled water, and rinses were collected. Filter membranes were then dried, insoluble fine particles trapped within the in-line filter were observed using a microscope, and trapped components were analyzed by Fourier transform-infrared spectroscopy (FTIR) (Spotlight 400, PerkinEImer Inc., Wellesley, MA, USA). As controls, 
in-line filters without CSTD use were collected after saline infusion. Additionally, materials such as rubber stoppers of anticancer drug vials, membranes of the connection part in CSTDs, and needles stored in CSTDs were analyzed as possible sources of contaminants by FTIR and scanning electron microscopy-energy dispersive Xray spectroscopy (SEM-EDS) (SEM: Quanta 200 FEG, FEI Company, Hillsboro, OR, USA; EDS: INCA Energy, OXFORD Inc., Oxford, UK) to clarify the insoluble fine particles trapped in in-line filters.

\section{Statistical analysis}

Statistical analyses were performed using SAS software package version 9.4 (SAS Institute Inc., Cary, NC, USA). Fisher's exact test was used for categorical data. P values $<0.05$ were considered indicative of statistical significance.

\section{Ethics of the Study}

This study was approved by the Ethics Committee for Clinical Studies at Ehime University Graduate School of Medicine (study \#1810019) and was carried out in accordance with the ethical standards of the 1995 Declaration of Helsinki (as revised in Brazil, 2013). Informed consent was by the opt-out principle. 


\section{References}

1 USP Pharmacopoeia Forum. In-Process Revision: <1790> VISUAL INSPECTION

OF INJECTIONS (Serial online); 1.

http://www.usppf.com/pf/pub/index.html (2020)

2 Brewer, J. H. \& Dunning, J. H. An in vitro and in vivo study of glass particles in ampules. J Am Pharm Assoc Am Pharm Assoc 36, 289-293 (1947).

3 Deluca, P. P., Rapp, R. P., Bivins, B., McKean, H. E. \& Griffen, W. O. Filtration and infusion phlebitis: a double-blind prospective clinical study. Am J Hosp Pharm 32, 1001-1007 (1975).

4 Schroeder, H. G. \& DeLuca, P. P. Particulate matter assessment of a clinical investigation on filtration and infusion phlebitis. Am J Hosp Pharm 33, 543-546 (1976).

5 Falchuk, K. H., Peterson, L. \& McNeil, B. J. Microparticulate-induced phlebitis. Its prevention by in-line filtration. N Engl J Med 312, 78-82, doi:10.1056/NEJM198501103120203 (1985). 
6 Kanke, M., Simmons, G. H., Weiss, D. L., Bivins, B. A. \& DeLuca, P. P. Clearance of 141C3-labeled microspheres from blood and distribution in specific organs following intravenous and intraarterial administration in beagle dogs. J Pharm Sci 69, 755-762, doi:10.1002/jps.2600690703 (1980).

7 Stehbens, W. E. \& Florey, H. W. The behavior of intravenously injected particles observed in chambers in rabbits' ears. Q J Exp Physiol Cogn Med Sci 45, 252264, doi:10.1113/expphysiol.1960.sp001470 (1960).

8 Shaw, N. J. \& Lyall, E. G. Hazards of glass ampoules. Br Med J (Clin Res Ed) 291, 1390, doi:10.1136/bmj.291.6506.1390 (1985).

9 Falck, K. et al. Mutagenicity in urine of nurses handling cytostatic drugs. Lancet 1, 1250-1251, doi:10.1016/s0140-6736(79)91939-1 (1979).

10 Rieche, K. Carcinogenicity of antineoplastic agents in man. Cancer Treat Rev 11, 39-67, doi:10.1016/0305-7372(84)90016-1 (1984).

11 Fairchild, W. V., Spence, C. R., Solomon, H. D. \& Gangai, M. P. The incidence of bladder cancer after cyclophosphamide therapy. J Urol 122, 163-164, doi:10.1016/s0022-5347(17)56306-5 (1979). 
12 Ratner, P. A. et al. Cancer incidence and adverse pregnancy outcome in registered nurses potentially exposed to antineoplastic drugs. BMC Nurs 9, 15 , doi:10.1186/1472-6955-9-15 (2010).

13 Skov, T. et al. Leukaemia and reproductive outcome among nurses handling antineoplastic drugs. Br J Ind Med 49, 855-861, doi:10.1136/oem.49.12.855 (1992).

14 Sessink, P. J., Boer, K. A., Scheefhals, A. P., Anzion, R. B. \& Bos, R. P. Occupational exposure to antineoplastic agents at several departments in a hospital. Environmental contamination and excretion of cyclophosphamide and ifosfamide in urine of exposed workers. Int Arch Occup Environ Health 64, 105112, doi:10.1007/BF00381477 (1992).

15 Ensslin, A. S. et al. Biological monitoring of cyclophosphamide and ifosfamide in urine of hospital personnel occupationally exposed to cytostatic drugs. Occup Environ Med 51, 229-233, doi:10.1136/oem.51.4.229 (1994).

16 Sessink, P. J., Van de Kerkhof, M. C., Anzion, R. B., Noordhoek, J. \& Bos, R. P. Environmental contamination and assessment of exposure to antineoplastic 
agents by determination of cyclophosphamide in urine of exposed pharmacy technicians: is skin absorption an important exposure route? Arch Environ Health 49, 165-169, doi:10.1080/00039896.1994.9940377 (1994).

17 Pethran, A. et al. Uptake of antineoplastic agents in pharmacy and hospital personnel. Part I: monitoring of urinary concentrations. Int Arch Occup Environ Health 76, 5-10, doi:10.1007/s00420-002-0383-8 (2003).

18 Connor, T. H., Anderson, R. W., Sessink, P. J. \& Spivey, S. M. Effectiveness of a closed-system device in containing surface contamination with cyclophosphamide and ifosfamide in an i.v. admixture area. Am J Health Syst Pharm 59, 68-72, doi:10.1093/ajhp/59.1.68 (2002).

19 Wick, C., Slawson, M. H., Jorgenson, J. A. \& Tyler, L. S. Using a closed-system protective device to reduce personnel exposure to antineoplastic agents. Am J Health Syst Pharm 60, 2314-2320, doi:10.1093/ajhp/60.22.2314 (2003).

20 Sessink, P. J., Connor, T. H., Jorgenson, J. A. \& Tyler, T. G. Reduction in surface contamination with antineoplastic drugs in 22 hospital pharmacies in the US 
following implementation of a closed-system drug transfer device. J Oncol Pharm Pract 17, 39-48 doi:10.1177/1078155210361431 (2011).

21 Clark, B. A. \& Sessink, P. J. Use of a closed system drug-transfer device eliminates surface contamination with antineoplastic agents. J Oncol Pharm Pract 19, 99-104, doi:10.1177/1078155212468367 (2013).

22 Harrison, B. R., Peters, B. G. \& Bing, M. R. Comparison of surface contamination with cyclophosphamide and fluorouracil using a closed-system drug transfer device versus standard preparation techniques. Am J Health Syst Pharm 63, 1736-1744, doi:10.2146/ajhp050258 (2006).

23 Garvan, J. M. \& Gunner, B. W. The harmful effects of particles in Intravenous fluids. Med J Aust 2, 1-6 (1964).

24 Sendo, T. et al. Particulate contamination of lyophilized amphotericin B preparation during reconstitution process. J Clin Pharm Ther 26, 87-91, doi:10.1046/j.1365-2710.2001.00328.x (2001). 
25 Trissel, L. A., Martinez, J. F. \& Simmons, M. Compatibility of etoposide phosphate with selected drugs during simulated Y-site injection. J Am Pharm Assoc (Wash) 39, 141-145, doi:10.1016/s1086-5802(16)30488-0 (1999).

26 British pharmacopoeia. https://www.pharmacopoeia.com/ (2020).

27 USP pharmacopoeia. https://www.usp.org/ (2020).

28 Japanese pharmacopoeia.

https://www.mhlw.go.jp/stf/seisakunitsuite/bunya/0000066530.html (2020).

29 Nakayama T, Shiotsuki A, Haruyama S, Sutou K, Tsuji Y. The handling devised to reduce the risk of leakage from rubber stopper at the time of preparation of anticancer drugs. JPHCS 39, 276-285, https://doi.org/10.5649/jjphcs.39.276 (2013).

30 Petoskey, F., Kwok, S. C., Jackson, W. \& Jiang, S. Overcoming Challenges of Implementing Closed System Transfer Device Clinical In-Use Compatibility Testing for Drug Development of Antibody Drug Conjugates. J Pharm Sci 109, 761-768, doi:10.1016 / j.xphs.2019.07.021 (2020). 
31 Sreedhara, A. et al. Challenges of Using Closed System Transfer Devices With Biological Drug Products: An Industry Perspective. J Pharm Sci 109, 22-29, doi:10.1016/j.xphs.2019.10.042 (2020). 


\section{Acknowledgments}

We thank Takatoshi Sakamoto (Matsuyama University, Japan) for performing experiments to identify crystals of oleic acid amides in the infusion route, and Tamae Yamamoto and Kengo Imai (Kobelco Research Institute, Inc., Japan) for conducting component analysis of insoluble particles.

\section{Disclosure Statement}

The authors have no conflict of interest.

\section{Author contributions statement}

SS and YY designed the study, collected clinical data, and drafted the manuscript. KA, TY, HH, CT, TN, TS, YH, YY, AT, NH and MT participated in study design and oversaw the clinical data. YY conceived the study, participated in its design and coordination, and helped to draft the manuscript. All authors read the draft, critically revised it, and approved the final manuscript. 


\section{Figure Legends}

Fig. 1. Frequencies of insoluble fine particles in preparation of monoclonal antibodies compared to those during preparation of cytotoxic agents at Ehime University Hospital. Others: all anticancer agents excluding monoclonal antibodies.

Fig. 2. Visual and structural analysis of insoluble fine particles using microscopy and Fourier transform-infrared spectroscopy (FTIR). (A) A polysaccharide containing a carboxylic acid structure was considered as a contaminant by FTIR analysis. (B) FTIR analysis indicated that polypropylene was considered as the main component, and fatty acids were considered as the secondary component. (C) FTIR analysis indicated that the main component was polypropylene, and the possibility of silicone as an accessory component was considered. 


\section{TABLE Legends}

Table 1. The manufacturers of generic drugs are listed in parentheses. Those without parentheses in the drug name mean the original product. Others: all anticancer agents excluding monoclonal antibodies.

Table 2. Frequencies of contaminations (insoluble particles) were calculated using the following formula: incidence of insoluble particles/total preparations. CSTD use rates were calculated using the following formula: CSTD use/total preparations (chemotherapies). CSTD: closed system drug transfer devices; Ehime Univ Hop: Ehime University Hospital.

Table 3. All agents are listed by ingredient name (original product); co-selling products of ingredients are described by product name. Manufacturers of generic drugs are listed in parentheses. NK, Nippon Kayaku Company, Limited (generic); MARUKO and Yakult, Yakult Company, Limited (generic); Nichi-Iko, Limited (generic); TEVE and Teva Takeda, Teva Takeda Pharma Company, Limited (generic); EE, Elmed Eisai and Nichi- 
Iko, Limited (generic); Nipro, Nipro Company, Limited (generic); Sandoz, Sandoz

Company, Limited (generic); TOWA, Towa Pharmaceutical Company, Limited (generic);

Hospira, Pfizer Company, Limited (generic); Sawai, Sawai Pharmaceutical Company,

Limited (generic); Taiho, Taiho Pharmaceutical Company, Limited (generic). Others: all anticancer agents excluding monoclonal antibodies. CSTD: closed system drug transfer devices. 


\section{Supporting information}

Table S1 shows the number of pharmacists and their years of experience with cancer chemotherapies to show the reliability of the current observation study. Ehime Univ Hop: Ehime University Hospital. 
Table 1. Incidence of insoluble fine particles during preparation of anticancer agents at

Ehime University Hospital (frequency = incident/preparation).

\begin{tabular}{|c|c|c|c|}
\hline Monoclonal antibodies & & & \\
\hline Agent & Incident $(\mathrm{n})$ & Frequency $(\mathrm{n})$ & Incident/Frequency $(\%)$ \\
\hline Pembrolizumab & 70 & 112 & 62.5 \\
\hline Ramucirumab & 36 & 75 & 48.0 \\
\hline Panitumumab & 18 & 40 & 45.0 \\
\hline Mogamulizumab & 2 & 5 & 40.0 \\
\hline Durvalumab & 3 & 9 & 33.3 \\
\hline Daratumumab & 19 & 57 & 33.3 \\
\hline Ipilimumab & 1 & 3 & 33.3 \\
\hline Cetuximab & 26 & 88 & 29.5 \\
\hline Bevacizumab & 140 & 763 & 18.3 \\
\hline Elotuzumab & 6 & 54 & 11.1 \\
\hline Brentuximab vedotin & 1 & 25 & 4.0 \\
\hline Pertuzumab & 3 & 89 & 3.4 \\
\hline Nivolumab & 1 & 34 & 2.9 \\
\hline Rituximab & 7 & 330 & 2.1 \\
\hline Aflibercept & 0 & 5 & 0.0 \\
\hline Obinutuzumab & 0 & 4 & 0.0 \\
\hline Atezolizumab & 0 & 25 & 0.0 \\
\hline Total & 333 & 1,718 & 19.4 \\
\hline
\end{tabular}

\begin{tabular}{|c|c|c|c|}
\hline Others & & & \\
\hline Agent & Incident $(\mathrm{n})$ & Frequency $(\mathrm{n})$ & Incident/ Frequency (\%) \\
\hline Pemetrexed & 16 & 34 & 47.1 \\
\hline Pralatrexate & 8 & 17 & 47.1 \\
\hline Vinorelbine & 1 & 6 & 16.7 \\
\hline Eribulin & 11 & 79 & 13.9 \\
\hline Cyclophosphamide & 32 & 271 & 11.8 \\
\hline Paclitaxel (NK) & 43 & 390 & 11.0 \\
\hline Carfilzomib & 1 & 18 & 5.6 \\
\hline Nab-paclitaxel & 9 & 257 & 3.5 \\
\hline Bendamustine & 2 & 72 & 2.8 \\
\hline Irinotecan & 2 & 205 & 1.0 \\
\hline Gemcitabine (Yakult) & 4 & 435 & 0.9 \\
\hline Doxorubicin (NK) & 1 & 214 & 0.5 \\
\hline Fluorouracil & 2 & 483 & 0.4 \\
\hline Nedaplatin & 0 & 2 & 0.0 \\
\hline Vinblastine & 0 & 53 & 0.0 \\
\hline Oxaliplatin & 0 & 262 & 0.0 \\
\hline Vincristine & 0 & 123 & 0.0 \\
\hline Amrubicin & 0 & 3 & 0.0 \\
\hline Carboplatin (NK) & 0 & 170 & 0.0 \\
\hline Cisplatin (MARUKO) & 0 & 88 & 0.0 \\
\hline Dacarbazine & 0 & 22 & 0.0 \\
\hline Liposomal doxorubicin & 0 & 10 & 0.0 \\
\hline Pirarubicin & 0 & 16 & 0.0 \\
\hline Total & 132 & 3,230 & 4.1 \\
\hline & & & \\
\hline & & & \\
\hline & 0 & 0.0 \\
\hline
\end{tabular}


Table 2. Summary of frequencies of contaminations at institutes providing

chemotherapies in Ehime Prefecture.

\begin{tabular}{|c|c|c|c|c|c|c|c|c|c|}
\hline \multirow{2}{*}{ Information of preparation } & \multicolumn{9}{|c|}{ Hospital } \\
\hline & Ehime Univ Hop & A & $\mathrm{B}$ & C & $\mathrm{D}$ & $\mathrm{E}$ & $\mathrm{F}$ & G & $\mathrm{H}$ \\
\hline CSTD type & A & A & A & A & $\mathrm{B}$ & $\mathrm{B}$ & B & $\mathrm{C}$ & C \\
\hline Preparations ( $\mathrm{n}$ ) & 1,818 & 4,920 & 669 & 5,701 & 158 & 585 & 205 & 638 & 2,677 \\
\hline Frequencies of CSTD use/total chemotherapies (\%) & 81.6 & 5.2 & 100 & 14.8 & 88.9 & 4 & 14.3 & 7.0 & 6.4 \\
\hline Incidence of contaminations ( $n$ ) & 167 & 2 & 1 & 14 & 0 & 0 & 0 & 0 & 16 \\
\hline Frequencies of contaminations (total) (\%) & 9.19 & 0.041 & 0.15 & 0.24 & 0 & 0 & 0 & 0 & 0.6 \\
\hline Frequencies of contaminations (CSTD use) (\%) & 10.8 & 0 & 0.2 & 0.8 & 0 & 0 & 0 & 0 & 0 \\
\hline Frequencies of contaminations (needle preparation) (\%) & 0 & 0.042 & - & 0.02 & 0 & 0 & 0 & 0 & 0.6 \\
\hline
\end{tabular}


Table 3. Incidence and frequency of contaminations during preparation of anticancer agents at institutes in Ehime Prefecture providing chemotherapies with or without CSTD use.

\begin{tabular}{|c|c|c|c|c|c|c|}
\hline \multicolumn{7}{|c|}{ CSTD use } \\
\hline & \multicolumn{3}{|c|}{ Yes } & \multicolumn{3}{|c|}{ No } \\
\hline Agent & Incident (n) & Frequency $(\mathrm{n})$ & Incident/ Frequency $(\%)$ & Incident $(\mathrm{n})$ & Frequency (n) & ncident/ Frequency (\%) \\
\hline Aflibercept & 3 & 3 & 100.0 & 0 & 44 & 0.0 \\
\hline Atezolizumab & 0 & 4 & 0.0 & 0 & 38 & 0.0 \\
\hline Bevacizumab & 26 & 273 & 9.5 & 0 & 994 & 0.0 \\
\hline Blinatumomab & 0 & 0 & - & 0 & 20 & 0.0 \\
\hline Brentuximab Vedotin & 0 & 9 & 0.0 & 0 & 6 & 0.0 \\
\hline Cetuximab & 5 & 13 & 38.5 & 0 & 101 & 0.0 \\
\hline Daratumumab & 6 & 17 & 35.3 & 1 & 97 & 1.0 \\
\hline Durvalumab & 2 & 13 & 15.4 & 0 & 161 & 0.0 \\
\hline Elotuzumab & 4 & 6 & 66.7 & 0 & 23 & 0.0 \\
\hline Herceptin & 0 & 20 & 0.0 & 2 & 782 & 0.3 \\
\hline Ipilimumab & 0 & 4 & 0 & 0 & 0 & \\
\hline Mogamulizumab & 2 & 6 & 33.3 & 0 & 4 & 0.0 \\
\hline Nivolumab & 1 & 107 & 0.9 & 0 & 442 & 0.0 \\
\hline Obinutuzumab & 0 & 15 & 0.0 & 0 & 40 & 0.0 \\
\hline Ofatumumab & 0 & 0 & - & 0 & 3 & 0.0 \\
\hline Panitumumab & 4 & 15 & 26.7 & 0 & 207 & 0.0 \\
\hline Pembrolizumab & 41 & 78 & $\begin{array}{l}2.1 \\
52.6\end{array}$ & 0 & 323 & 0.0 \\
\hline Pertuzumab & $\begin{array}{c}41 \\
0\end{array}$ & 25 & 0.0 & 0 & 239 & 0.0 \\
\hline Ramucirumab & 14 & 62 & 22.6 & 0 & 231 & 0.0 \\
\hline Rituximab & 4 & 58 & 6.9 & 0 & 124 & 0.0 \\
\hline Rituximab(KHK) & 1 & 4 & $\begin{array}{l}25.0 \\
25.0\end{array}$ & 0 & 170 & 0.0 \\
\hline Trastuzumab Emtansine & 0 & 0 & 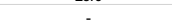 & 0 & 64 & 0.0 \\
\hline Total & 113 & 732 & 15.4 & 3 & 4,113 & 0.073 \\
\hline
\end{tabular}

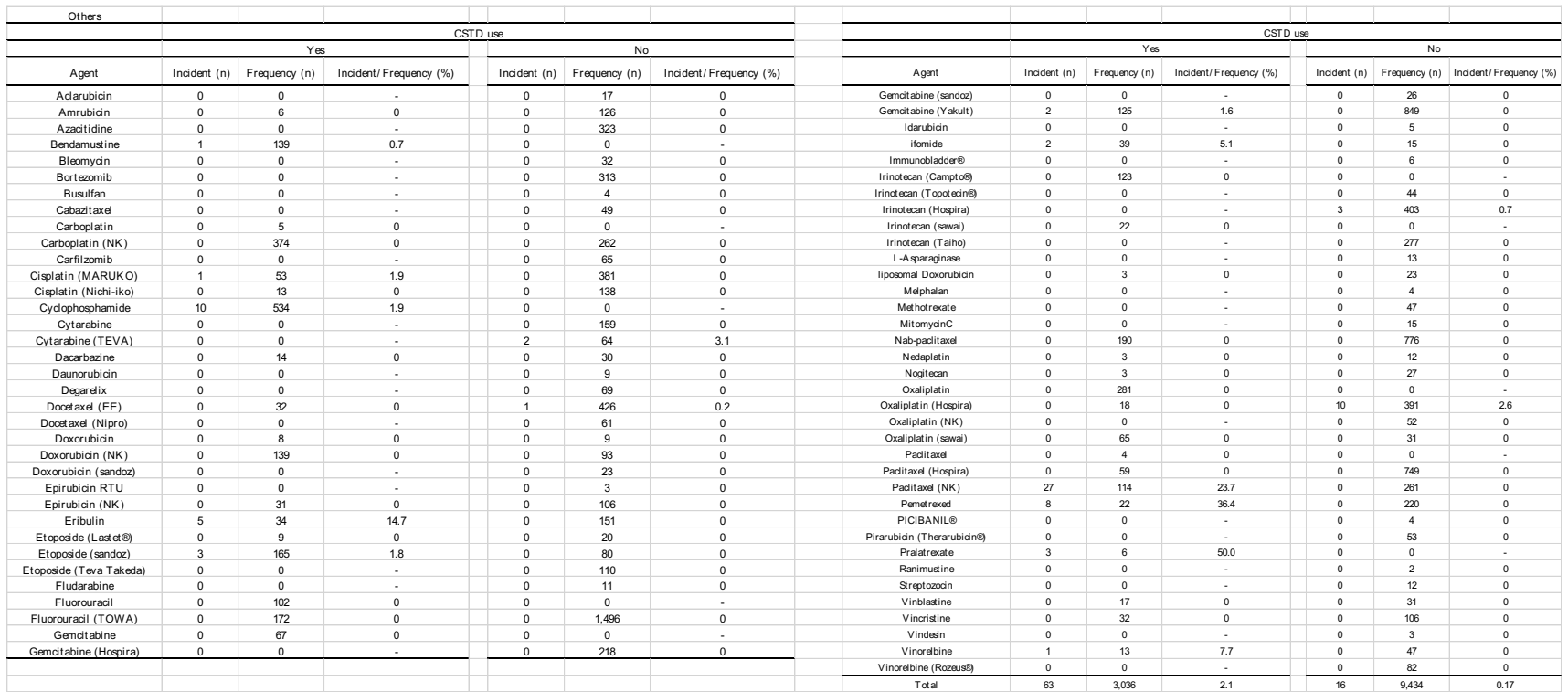


Figures

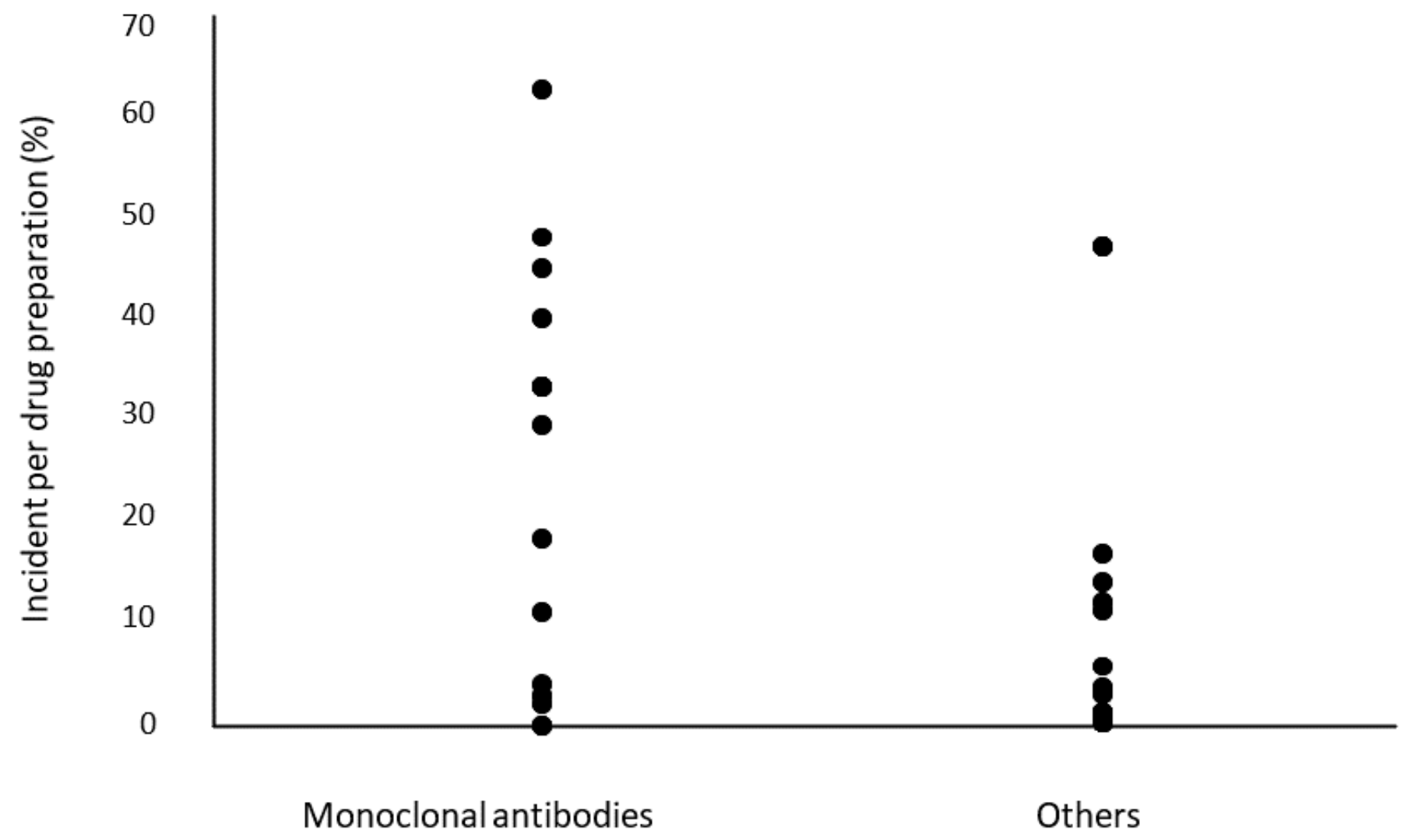

Figure 1

Frequencies of insoluble fine particles in preparation of monoclonal antibodies compared to those during preparation of cytotoxic agents at Ehime University Hospital. Others: all anticancer agents excluding monoclonal antibodies. 


\section{(A)}
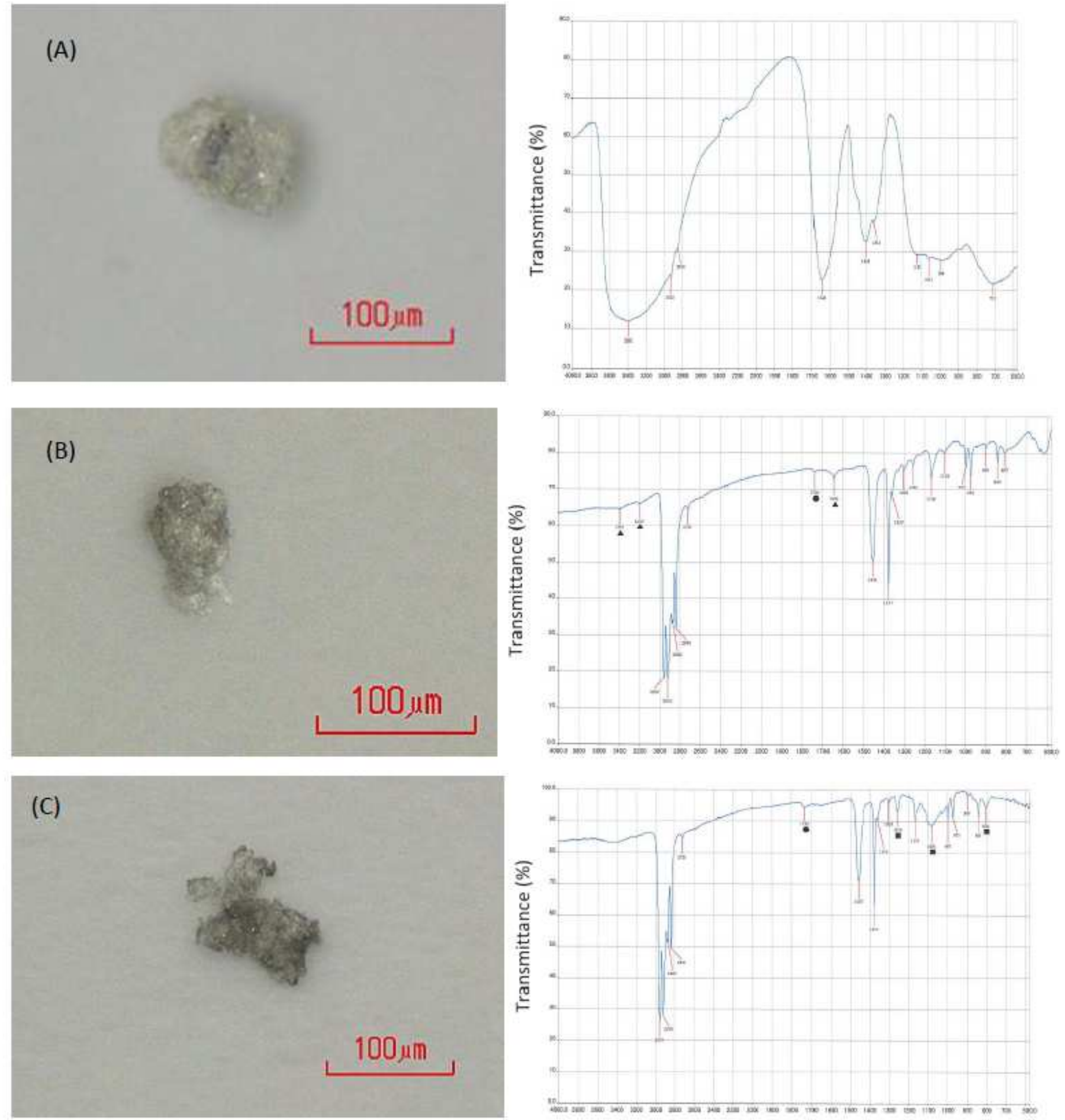

\section{Figure 2}

Visual and structural analysis of insoluble fine particles using microscopy and Fourier transform-infrared spectroscopy (FTIR). (A) A polysaccharide containing a carboxylic acid structure was considered as a contaminant by FTIR analysis. (B) FTIR analysis indicated that polypropylene was considered as the main component, and fatty acids were considered as the secondary component. (C) FTIR analysis 
indicated that the main component was polypropylene, and the possibility of silicone as an accessory component was considered.

\section{Supplementary Files}

This is a list of supplementary files associated with this preprint. Click to download.

- Supportinginformation.docx 